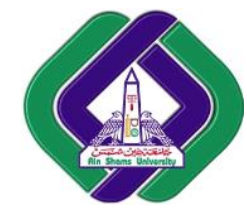

International Journal of Intelligent Computing and Information Science

\title{
DETECTING AND RESOLVING AMBIGUITY APPROACH IN REQUIREMENT SPECIFICATION: IMPLEMENTATION, RESULTS AND EVALUATION
}

\author{
Somaia Osama \\ Mostafa Aref
Computer Science Department, Faculty of Computer and Information Science, Ain Shams University Cairo, Egypt
somaia.osama.r@gmail.com
aref_99@yahoo.com

\begin{abstract}
Requirements documentsare always written in natural language. At the point when a sentence can be understood diversely among various readersambiguity is happened [1]. In this paper, we illustrate an automated tool for detectingand resolvingambiguities thatcause a high risk of misunderstanding byseveralreaders and lead to confusion, waste of both effort and time and rework. Sentences in a natural language requirements specification document thathaveambiguity are initialdetected automatically from the text andambiguity type is determined. Sentences thatincludeambiguity are thenresolved automatically also by resolving algorithm based on a set of rules that we collected from training data. We implemented a tool for Detecting and Resolving Ambiguity (DARA), in order to clarifyand estimate our approach. The tool focuses on Lexical, Referential, Coordination, Scope and Vague ambiguity. We determine on the results of a collection of requirement specification documents to evaluatethe performance and utility of the approach.
\end{abstract}

Keywords: Ambiguity, ambiguity detection, ambiguity resolving, disambiguation, natural language processing $(N L P)$, requirement engineering, software requirement specification,

\section{Introduction}

The Software Requirements Specification (SRS) is a part of the contract and it must define the user and the system requirements clearly, precisely and unambiguously [2]. The SRS that has inconspicuous, incomplete, unmanaged, unspecified, inaccurate or ambiguous requirement definition may eventually lead to cost and time overruns [3, 4, and 5]. Ambiguity is the possibility to understand a phrase or word in different ways. It is one of the issues that happen in natural language documents. An ambiguity has two sources: communication faultsand inadequate information. Some errors can be resolved without domain knowledge like grammatical error, though some errors need domain knowledge like the lack of details that the user needs. The Ambiguity Handbook [6] presents different types of ambiguities, categorized as Lexical, Syntactic, Semantic, Pragmatic, Vagueness, Generality and Language Error. Although the fact that the 
requirements specified in natural language tend to inappropriate interpretations, the requirements are most often specified in natural language[7, 8]. So, it is necessary to develop the approaches whichhandle the ambiguities in user requirement specifications. Manually detecting and resolving ambiguity from software requirements is a boring, time consuming, cause errors, and therefore expensive process. So, an approach to detect and resolve ambiguities automaticallyfrom the requirements statement is needed.

\section{DARA Architecture}

This section provides an architectural description of the DARA system. It was developed to be modular, extensible, and simple to utilize. We develop an automated system to detect and resolve ambiguities from full text documents. The DARA architecture is shown in Figure 1. The initial input is a complete requirement text. The output is unambiguous requirement texts.

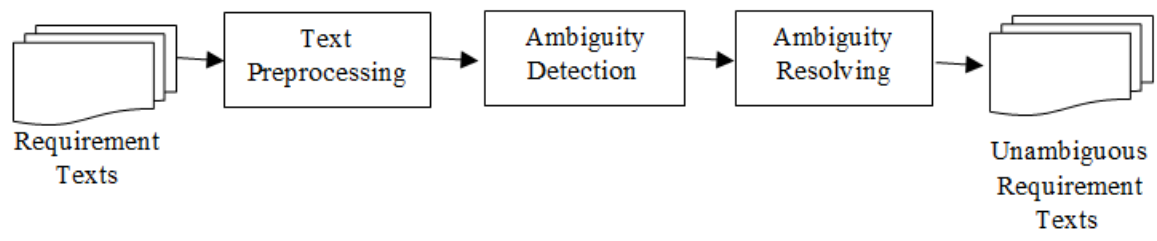

Figure 1DARAArchitecture

The system consists of three major functional process modules

\subsection{The Text Preprocessing Module}

The Text preprocessing module consists of four stages as shown in Figure 2.

- Sentence splitter: Each sentence is isolatedfrom the input text and is returnedasset of strings.

- Tokenizer: Each sentence is capturedas an input and is separatedinto tokens for examplewords, numbers and punctuation.

- Parts of speech (POS tagger):The words in a documentare determined to a specific part of speech.

- Syntactic parser: sequences of words are changed into structures that show how the sentence's partsconnect to each other. This phaseassists us in recognizing the fundamental parts in each sentence such as subject, object, verb...etc[9].

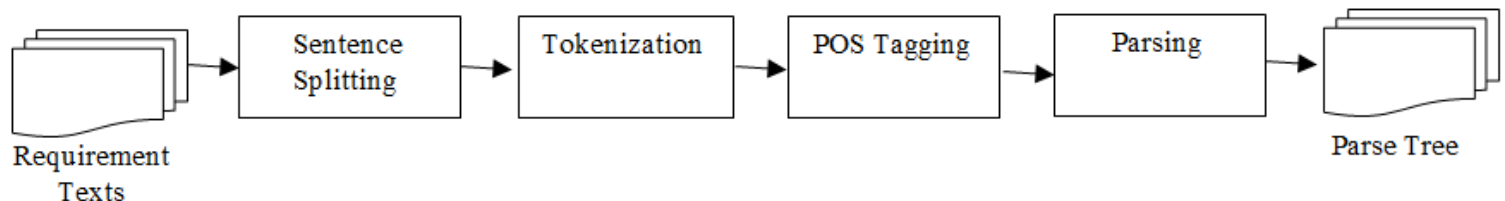

Figure 2 The Text Preprocessing Module

\subsection{The Ambiguity Detection Module}

This module could apply a several ambiguity measures to a requirement specification to recognize possibly ambiguous sentences. The core goals for this tool are: to detect which sentences in a natural language requirement specification are ambiguous and, for each ambiguous sentence, identify the ambiguity word 
and ambiguity type. And calculate the percentage of each ambiguity typein the document. Figure 3 shows The Ambiguity Detection Module architecture.

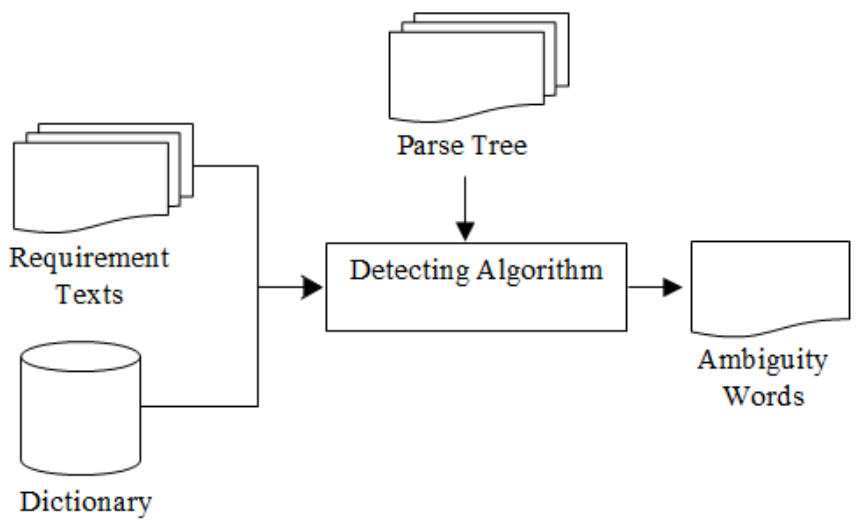

Figure 3The Ambiguity Detection Module

Dictionary is the fundamental element of ambiguity detection whichcontains the ambiguity indicators[10]in the documents. Ambiguous words that outcome from misinterpreted requirements are analyzed and saved into the dictionary. The major goal of this phase is to check and seeif the wordsin software requirements specification document are ambiguous or unambiguous. There are five types of ambiguity Lexical Ambiguity, Referential Ambiguity, Coordination Ambiguity, Scope Ambiguity, Vague. So we identify indicators of each type.

i. Identify Lexical Ambiguity

The Lexical dictionary contains the possible ambiguity indicators such as: bound, break, call, content, continue, contract, count, direct, even, express, form, forward, function, get, job, level, name, notice, number, out, part, position, record, reference, return, set, source, special, standard, string, subject, switch, tail, throw, throw, throw, translate, try, under, value ,and way.

ii. Identify Referential Ambiguity

The Referential dictionarycontains the possible ambiguity indicators such as: I, he, she,it, me, her, him,them,hers, his, its, your, their, our,herself, himself, itself, ours, ourselves, yourself,themselves,yourselves, that, theirs, these, they, this, which, who, you, yours, someone,anyone, everyone,somebody, anybody,everybody,something, anything, and everything.

iii. Identify Coordination Ambiguity

The Coordination dictionarycontains the possible ambiguity indicators such as: and also, and, and/or, but, if and only if, if then, or, and unless.

iv. Identify Scope Ambiguity

The Scope dictionarycontains the possible ambiguity indicators such as: a, all, any, each, few, little, many, much, not, several, and some. 


\section{v. Identify Vague}

The Vague dictionary contains the possible ambiguity indicators such as: available, common, capability, consistent, easily, easy, effective, efficient, full, general, maximum, minimum, powerful, particular, quickly, random, recent, sufficient, sufficiently, sequential, significant, simple, useful, and various.

\subsection{The Ambiguity Resolving Module}

Finally, this module focuses in removing and resolving the ambiguity. For each ambiguous sentence, resolve the ambiguity in the sentence automatically as the final step using resolving rules, and therefore improve the natural language requirement specificationdocument. Figure 4 shows The Ambiguity Resolving Module architecture.

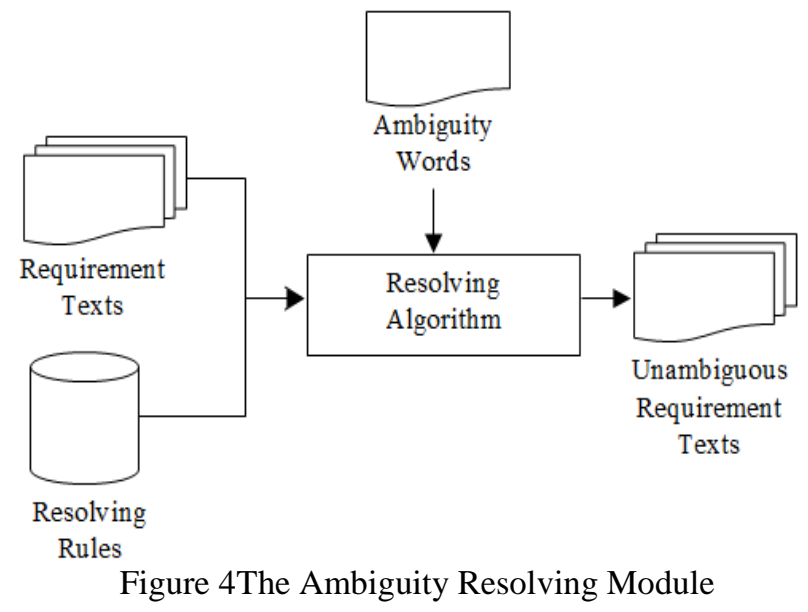

The resolving ambiguity approach uses the following common rules to check if a sentence contains an ambiguity:

$\underline{\text { Rule 1: }}$ when sentence containing not only, but also, as well as, both, but, and, and also, or, and/or,X/Y, either, whether, otherwise, meanwhile, whereas, on the other hand split it to two sentences.

Rule 2: when sentence containing unless, replace with if not.

Rule 3: when sentence containing a, an, all, any, some, every, several replace with each.

Rule 4: when sentence containing should, will, would, may, might, ought to replace with shall.

Rule 5: when sentence containing There is $\mathbf{X}$ in $\mathbf{Y}, \mathbf{X}$ exists in $\mathbf{Y}$ replace with $\mathbf{Y}$ has $\mathbf{X}$.

Rule 6: when sentence containing anaphora or pronoun such as they or them replaces with the farthest noun.

Rule 7: when sentence containing that replace with each of which.

Rule 8: when sentence containing only, also, almost, even, hardly, just, merely, nearly, and really put itafterthe first verb.

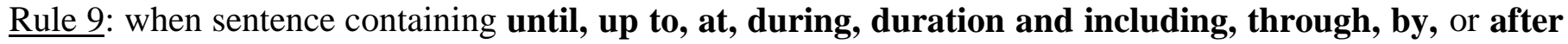
add only before it.

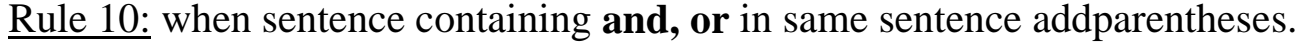


Rule 11:when sentence containing many replace with each of many.

Rule 12: when sentence containing few replace with each of few.

Rule 13: when sentence containing for up to replace with for up to and including.

Rule 14: when sentence containing plural nouns add each before it.

\section{DARA implementation, results and analysis}

DARA was developed using the openNLP and Java language. The Apache OpenNLP library is aJava libraryopen source and machine learning depend on toolkit for the handling of natural language document.OpenNLPsupportsNLP services like sentence segmentation,tokenization, part of speech tagging, parsing, chunking, named entity extraction, and coreference resolution. These services are required to implement more advanced text processing tasks. The OpenNLP library was used to build an effective text processing service. In this section the screenshot of DARA is provided. The graphical user interface is shown to facilitate in the description. Figure 5 show the GUI when the tool is in the run state. The DARA GUI is composed of four principal windows:

- Input Window - shows the content of the document file containing the requirements to be detected ambiguity and resolved and analyzed.

- Output Window - showsthe detected and resolved software requirement specification.

- Dictionary Window-shows the content of the dictionaries and contains function buttons for dictionary handling.

- Analysis Window-shows total ambiguities present in the software requirement specification document and percentage ofall ambiguity types and graphical representation.

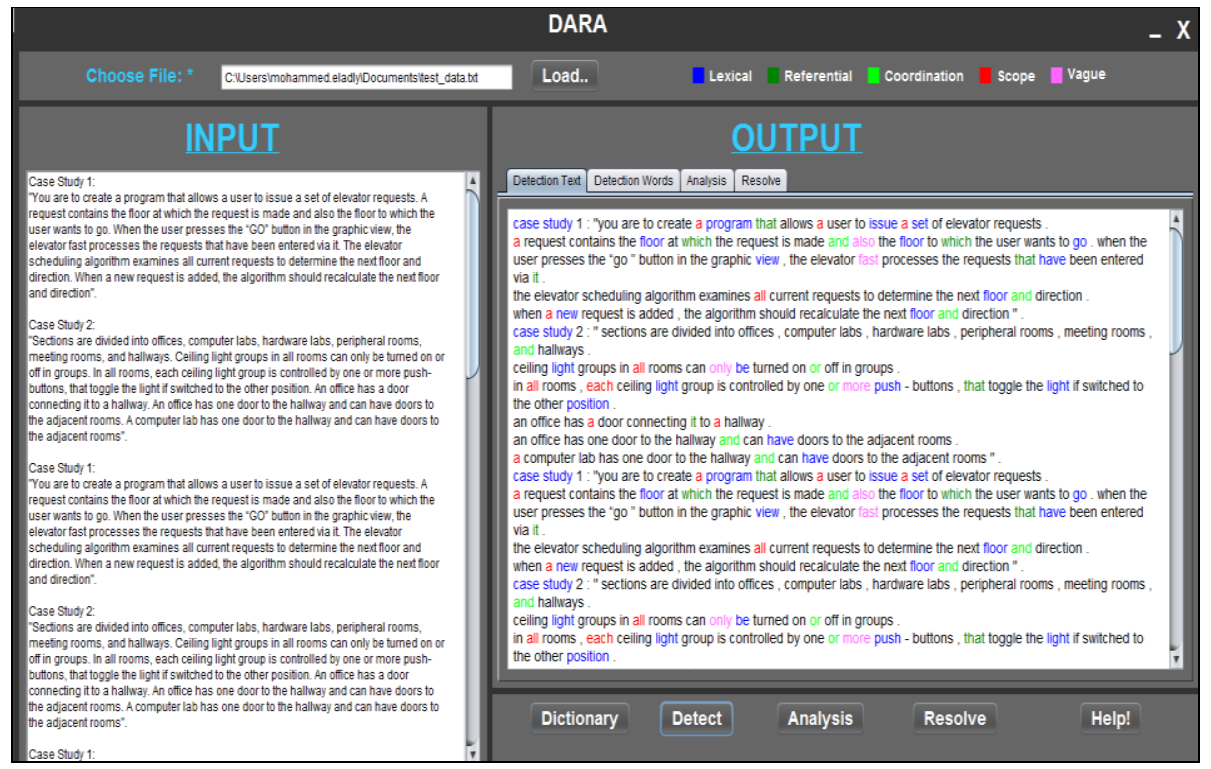

\subsection{Inputs Data}

Figure 5 DARA User Interface 
An analysis of real requirement documents taken from industrial software projects was performed by DARA, in order to test the tool and understand if it may provide a real support to the improvement of the quality of natural language requirements in an industrial environment. The requirement specification documents that were analyzed come from different application domains. The total set of software requirement specification documents is composed of 36 items. The requirements documents were collected from different websites. Number of lines and source of sample software requirement specification documents are presented in Table1.

Table 1Requirements Specification Documents Details

\begin{tabular}{|c|c|c|c|}
\hline ID & Title & \#Sentences & Link \\
\hline D1 & ECMA Standard ECMA-262 & 34961 & http://www.ecma-international.org \\
\hline $\mathrm{D} 2$ & FlexRay ${ }^{T M}$ & 17133 & $\underline{\text { https://svn.ipd.kit.edu }}$ \\
\hline D3 & Landsat Processing System & 14726 & $\underline{\text { http://research.it.uts.edu.au }}$ \\
\hline D4 & The investigation and control of outbreaks & 9440 & http://goo.gl/Sv4Ebu \\
\hline D5 & German Health Professional Card & 9086 & http://www.dkgev.de \\
\hline D6 & Joint Mapping Toolkit & 7341 & $\underline{\text { http://research.it.uts.edu.au }}$ \\
\hline D7 & VoteCal & 3070 & http://elections.cdn.sos.ca.gov \\
\hline D8 & Communication Services for DII & 2749 & $\underline{\text { http://research.it.uts.edu.au }}$ \\
\hline D9 & Foodborn outbreak management & 2355 & http://goo.gl/pTlgp9 \\
\hline D10 & Outbreak management guidelines for healthcare & 2099 & http://goo.gl/EcYVEi \\
\hline D11 & WHO guidelines for epidemic preparedness & 2094 & http://goo.gl/PK9yn7 \\
\hline D12 & Application to clinical and Public Health & 1885 & http://goo.gl/hVVy1Y \\
\hline D13 & Document for the Labor Market Information & 1856 & http://research.it.uts.edu.au \\
\hline D14 & SplitPay & 1573 & https://www.cise.ufl.edu \\
\hline D15 & Pacemaker & 1378 & https://svn.ipd.kit.edu \\
\hline D16 & A-7E Avionics System & 1339 & https://svn.ipd.kit.edu \\
\hline D17 & MODIS Science Data Processing Software & 1117 & http://research.it.uts.edu.au \\
\hline D18 & PHEMCE strategy & 1064 & http://goo.gl/hYaipm \\
\hline D19 & Civil Protection Service Resource System & 1014 & https://svn.ipd.kit.edu \\
\hline D20 & Defense Information Infrastructure & 769 & http://research.it.uts.edu.au \\
\hline D21 & Coincidence Matrix in the ATLAS Muon & 596 & $\underline{\text { http://research.it.uts.edu.au }}$ \\
\hline D22 & Post Grass System & 516 & http://research.it.uts.edu.au \\
\hline D23 & Light Control System & 427 & http://research.it.uts.edu.au \\
\hline D24 & E-Store Project & 419 & https://www.utdallas.edu \\
\hline D25 & University Library Information System & 408 & https://svn.ipd.kit.edu \\
\hline D26 & Developing a management system & 401 & http://goo.gl/015sth \\
\hline D27 & Ludo & 398 & $\underline{\text { https://svn.ipd.kit.edu }}$ \\
\hline D28 & Whois Protocol & 187 & http://www.ietf.org \\
\hline D29 & Display Management System & 91 & https://svn.ipd.kit.edu \\
\hline D30 & Cable TV Package Purchase & 79 & https://svn.ipd.kit.edu \\
\hline D31 & ATM Simulation & 33 & http://nlrp.ipd.kit.edu \\
\hline D32 & Sogno Hotel Reservation Service & 24 & https://svn.ipd.kit.edu \\
\hline D33 & Library & 18 & $\underline{\text { http://nlrp.ipd.kit.edu }}$ \\
\hline D34 & Ambulance Despatching System & 17 & $\underline{\text { https://svn.ipd.kit.edu }}$ \\
\hline D35 & Address Book & 14 & http://nlrp.ipd.kit.edu \\
\hline D36 & Mellor's Steam Boiler & 7 & http://nlrp.ipd.kit.edu \\
\hline
\end{tabular}




\subsection{Outputs Data}

The results of this type of validation have been very interesting. They are presented in the Table 2 that shows, for each evaluated document, the number of indicators' occurrencesof all the datasets. It displays the total numbers of ambiguities occur in software requirement specification documents and percentages of lexical,referential, coordination,scope and vague ambiguity for each document of software requirement specification. All experiments were executed on a $2.3 \mathrm{GHz}$ Intel Core i5 with $4 \mathrm{~GB}$ of memory.

Table 2The Occurrences of The Possible Ambiguities for each Indicator

\begin{tabular}{|c|c|c|c|c|c|c|c|c|}
\hline ID & $\begin{array}{c}\text { Number } \\
\text { of Lexical } \\
\text { Ambiguity }\end{array}$ & $\begin{array}{l}\text { Number of } \\
\text { Referential } \\
\text { Ambiguity }\end{array}$ & $\begin{array}{c}\text { Number of } \\
\text { Coordination } \\
\text { Ambiguity }\end{array}$ & $\begin{array}{c}\text { Number } \\
\text { of Scope } \\
\text { Ambiguity }\end{array}$ & $\begin{array}{c}\text { Number } \\
\text { of Vague } \\
\text { Ambiguity }\end{array}$ & $\begin{array}{l}\text { Number of } \\
\text { Detected } \\
\text { Sentences }\end{array}$ & $\begin{array}{l}\text { Number of } \\
\text { Resolved } \\
\text { Sentences }\end{array}$ & Time \\
\hline D1 & $38.8 \%$ & $12.7 \%$ & $8.8 \%$ & $20.6 \%$ & $19.1 \%$ & $56.4 \%$ & $44.6 \%$ & $24 \mathrm{~min}$ \\
\hline D2 & $37.6 \%$ & $11.8 \%$ & $10.8 \%$ & $19.6 \%$ & $20.3 \%$ & $58.4 \%$ & $55.6 \%$ & $8 \mathrm{~min}$ \\
\hline D3 & $47.8 \%$ & $10.9 \%$ & $14.9 \%$ & $13.4 \%$ & $13.1 \%$ & $35.8 \%$ & $46.2 \%$ & $4 \mathrm{~min}$ \\
\hline D4 & $22.8 \%$ & $14.2 \%$ & $19.5 \%$ & $16.9 \%$ & $26.5 \%$ & $65.2 \%$ & $62.3 \%$ & $4 \mathrm{~min}$ \\
\hline D5 & $44.0 \%$ & $8.1 \%$ & $13.8 \%$ & $13.1 \%$ & $21.0 \%$ & $36.5 \%$ & $42.7 \%$ & $4 \mathrm{~min}$ \\
\hline D6 & $33.7 \%$ & $7.5 \%$ & $19.6 \%$ & $20.2 \%$ & $19.0 \%$ & $32.8 \%$ & $65.0 \%$ & $2 \mathrm{~min}$ \\
\hline D7 & $36.0 \%$ & $13.0 \%$ & $12.2 \%$ & $11.7 \%$ & $27.1 \%$ & $38.3 \%$ & $50.3 \%$ & $1 \mathrm{~min}$ \\
\hline D8 & $43.2 \%$ & $7.5 \%$ & $18.6 \%$ & $14.2 \%$ & $16.4 \%$ & $49.2 \%$ & $49.9 \%$ & $1 \mathrm{~min}$ \\
\hline D9 & $20.7 \%$ & $13.0 \%$ & $22.3 \%$ & $14.3 \%$ & $29.7 \%$ & $68.2 \%$ & $73.4 \%$ & $1 \mathrm{~min}$ \\
\hline D10 & $24.3 \%$ & $12.3 \%$ & $22.4 \%$ & $16.8 \%$ & $24.2 \%$ & $72.3 \%$ & $73.4 \%$ & $1 \mathrm{~min}$ \\
\hline D11 & $22.4 \%$ & $13.0 \%$ & $21.6 \%$ & $17.8 \%$ & $25.2 \%$ & $59.2 \%$ & $69.9 \%$ & $1 \mathrm{~min}$ \\
\hline D12 & $25.1 \%$ & $14.2 \%$ & $22.5 \%$ & $14.8 \%$ & $23.4 \%$ & $57.2 \%$ & $57.8 \%$ & $53 \mathrm{sec}$ \\
\hline D13 & $28.1 \%$ & $9.4 \%$ & $20.8 \%$ & $13.7 \%$ & $28.0 \%$ & $76.8 \%$ & $83.6 \%$ & $55 \mathrm{sec}$ \\
\hline D14 & $28.2 \%$ & $16.0 \%$ & $13.4 \%$ & $21.7 \%$ & $20.8 \%$ & $52.3 \%$ & $67.4 \%$ & $32 \mathrm{sec}$ \\
\hline D15 & $36.4 \%$ & $7.9 \%$ & $18.3 \%$ & $16.8 \%$ & $20.6 \%$ & $43.0 \%$ & $58.8 \%$ & $36 \mathrm{sec}$ \\
\hline D16 & $28.9 \%$ & $23.2 \%$ & $12.4 \%$ & $18.1 \%$ & $17.4 \%$ & $65.0 \%$ & $61.4 \%$ & $34 \mathrm{sec}$ \\
\hline D17 & $40.7 \%$ & $4.3 \%$ & $19.7 \%$ & $8.7 \%$ & $26.7 \%$ & $47.9 \%$ & $38.9 \%$ & $48 \mathrm{sec}$ \\
\hline D18 & $21.4 \%$ & $14.4 \%$ & $29.7 \%$ & $9.0 \%$ & $25.6 \%$ & $63.9 \%$ & $75.0 \%$ & $50 \mathrm{sec}$ \\
\hline D19 & $23.8 \%$ & $17.3 \%$ & $15.2 \%$ & $19.8 \%$ & $24.0 \%$ & $73.1 \%$ & $62.6 \%$ & $46 \mathrm{sec}$ \\
\hline D20 & $26.8 \%$ & $14.4 \%$ & $22.2 \%$ & $18.7 \%$ & $17.9 \%$ & $51.4 \%$ & $67.6 \%$ & $52 \mathrm{sec}$ \\
\hline D21 & $38.3 \%$ & $10.6 \%$ & $11.7 \%$ & $12.0 \%$ & $27.5 \%$ & $55.4 \%$ & $50.3 \%$ & $30 \mathrm{sec}$ \\
\hline D22 & $30.2 \%$ & $12.2 \%$ & $15.0 \%$ & $18.2 \%$ & $24.4 \%$ & $57.0 \%$ & $54.4 \%$ & $19 \mathrm{sec}$ \\
\hline D23 & $47.1 \%$ & $9.3 \%$ & $9.7 \%$ & $21.1 \%$ & $12.8 \%$ & $65.6 \%$ & $62.9 \%$ & $23 \mathrm{sec}$ \\
\hline D24 & $31.9 \%$ & $16.8 \%$ & $18.0 \%$ & $15.1 \%$ & $18.3 \%$ & $39.1 \%$ & $58.5 \%$ & $18 \mathrm{sec}$ \\
\hline D25 & $29.7 \%$ & $16.8 \%$ & $13.3 \%$ & $18.7 \%$ & $21.4 \%$ & $61.0 \%$ & $55.8 \%$ & $19 \mathrm{sec}$ \\
\hline D26 & $20.5 \%$ & $31.0 \%$ & $18.0 \%$ & $11.6 \%$ & $18.9 \%$ & $80.5 \%$ & $73.1 \%$ & $30 \mathrm{sec}$ \\
\hline D27 & $43.7 \%$ & $13.2 \%$ & $6.8 \%$ & $21.6 \%$ & $14.7 \%$ & $18.3 \%$ & $53.4 \%$ & $17 \mathrm{sec}$ \\
\hline D28 & $18.9 \%$ & $21.4 \%$ & $19.9 \%$ & $20.9 \%$ & $18.9 \%$ & $51.3 \%$ & $66.7 \%$ & $40 \mathrm{sec}$ \\
\hline D29 & $26.7 \%$ & $16.3 \%$ & $23.0 \%$ & $12.6 \%$ & $21.5 \%$ & $54.9 \%$ & $72.0 \%$ & $17 \mathrm{sec}$ \\
\hline D30 & $24.2 \%$ & $17.5 \%$ & $11.7 \%$ & $17.5 \%$ & $29.2 \%$ & $51.9 \%$ & $82.9 \%$ & $22 \mathrm{sec}$ \\
\hline D31 & $23.5 \%$ & $8.2 \%$ & $12.4 \%$ & $30.0 \%$ & $25.9 \%$ & $84.8 \%$ & $96.4 \%$ & $32 \mathrm{sec}$ \\
\hline D32 & $27.9 \%$ & $17.6 \%$ & $11.8 \%$ & $26.5 \%$ & $16.2 \%$ & $100.0 \%$ & $79.2 \%$ & $28 \mathrm{sec}$ \\
\hline D33 & $28.8 \%$ & $1.7 \%$ & $20.3 \%$ & $39.0 \%$ & $10.2 \%$ & $88.9 \%$ & $100.0 \%$ & $12 \mathrm{sec}$ \\
\hline D34 & $13.7 \%$ & $9.8 \%$ & $5.9 \%$ & $47.1 \%$ & $23.5 \%$ & $76.5 \%$ & $84.6 \%$ & $18 \mathrm{sec}$ \\
\hline
\end{tabular}




\begin{tabular}{|l|l|l|l|l|l|l|l|l|}
\hline D35 & $40.8 \%$ & $9.9 \%$ & $12.0 \%$ & $21.8 \%$ & $15.5 \%$ & $85.7 \%$ & $100.0 \%$ & $22 \mathrm{sec}$ \\
\hline D36 & $45.0 \%$ & $2.5 \%$ & $12.5 \%$ & $27.5 \%$ & $12.5 \%$ & $100.0 \%$ & $100.0 \%$ & $25 \mathrm{sec}$ \\
\hline
\end{tabular}

Figure 6 shows that some particular ambiguities are more frequently detected than others by DARA. Especially lexical, scope and vague ambiguity seems to be the types of ambiguityimpacting in a large part of the requirement sentences of documents. Figure 6 shows that document 3 demonstrate a decrease in percentage distribution of all ambiguity types detected because of the document domain(Document 3 about satellite) and it shows that document 26 demonstrate an increase in percentage distribution of all ambiguity types detected because of the document domain covered in dictionaries.
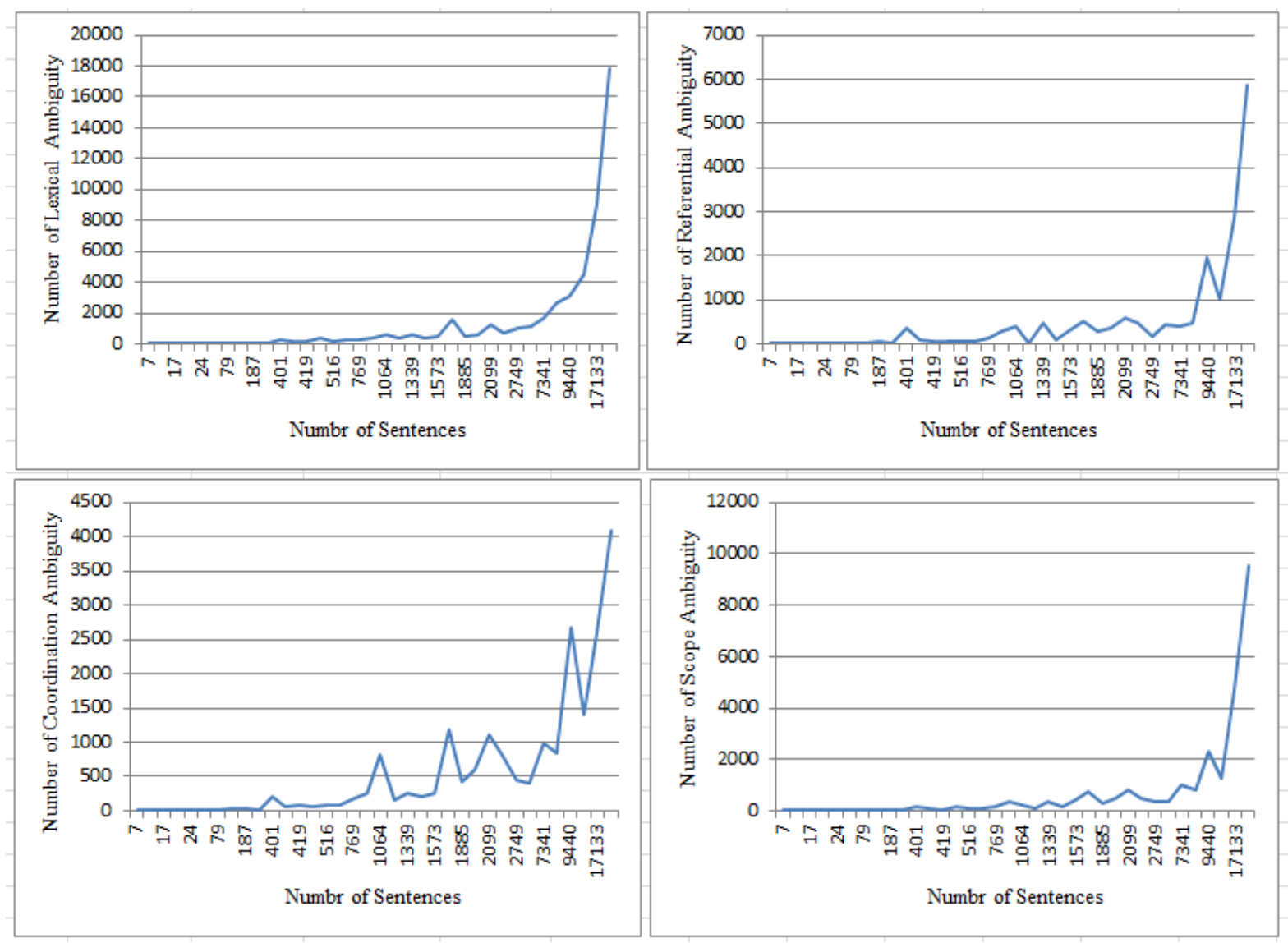


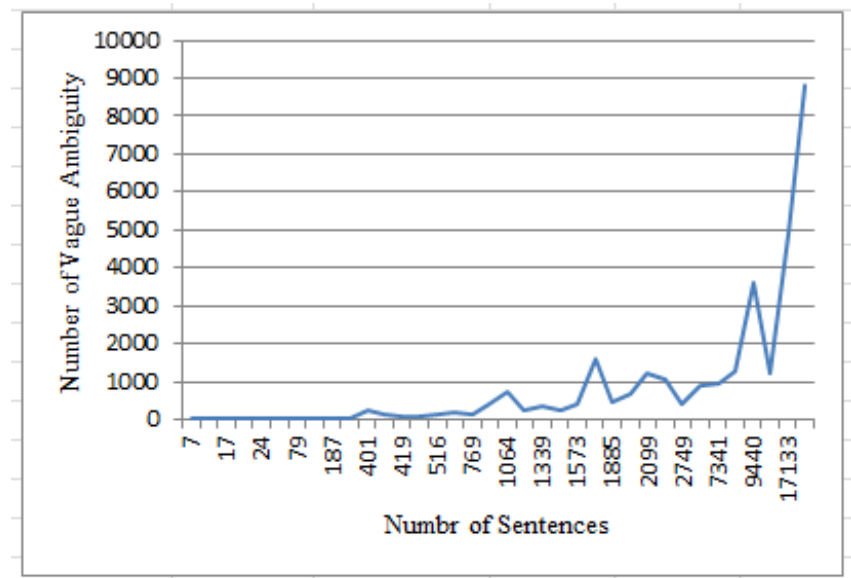

Figure 6 Percentage distribution of all ambiguity types detected

Figure 7 shows the percentage of each type of ambiguities is detected by DARA.The outcomes of the use of DARA on these 36 case studies show that the occurrences of the possible ambiguities are significantly high around $60 \%$ of the total number of requirements sentences (lexical ambiguity $37 \%$, referential ambiguity $9 \%$, coordination ambiguity 13\%, scope ambiguity $25 \%$ and vague 16\%). Figure 8 shows the numbers of detected and resolved sentences by DARA. The outcomes of the use of DARA on these 36 case studies show that the number of detected sentences that have possible ambiguities and the number of solved sentences are increased when the number of sentences increased. DARA solve $67 \%$ of ambiguities in the total number of requirements sentences.

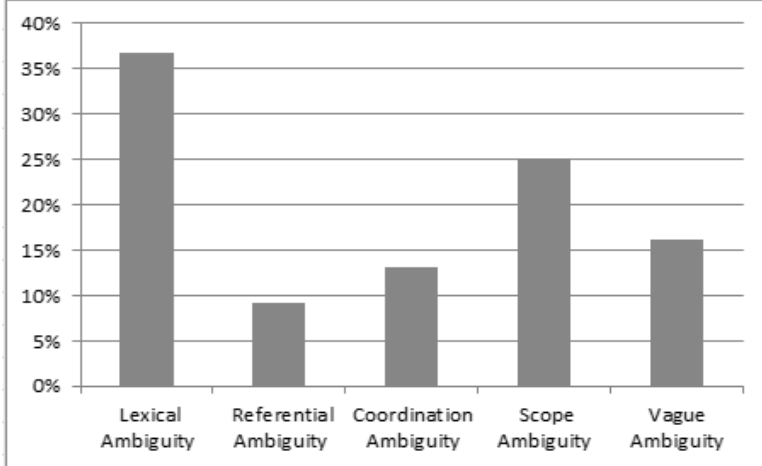

Figure 7 Percentage distribution of ambiguity types detected

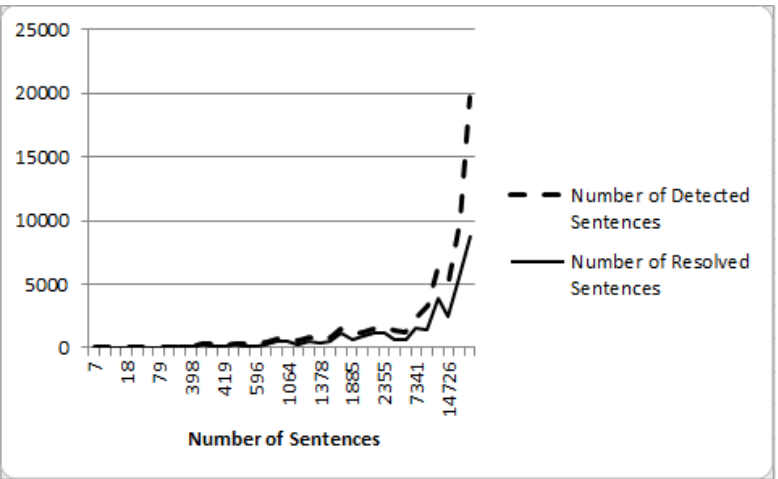

Figure 8 Percentage distributions of detected and resolved sentences

\section{Conclusion}

We have discussed that ambiguity is common in natural language requirements. When different stakeholders understand the same text differently, system incorrectly implementedriskbeing high [11]. So we implement DARA to detect and resolve ambiguities.According to the defined approach, DARA doesn't force the requirement engineers to follow a particular standard or style in writing [12].We execute our approach on 36 case studies. DARA can detect lexical ambiguity, referential ambiguity, coordination ambiguity, scope ambiguity, vague. It can measure the percentage of each ambiguity type. It can solve $67 \%$ of ambiguities in NL requirements specification documents. In this paper,by using a rule based approach 
we proved that it is possible to identify and resolve ambiguity automatically in natural language requirements. We employed algorithm to recognize ambiguities from sentences using dictionaries. Our aim is to enhance the requirements quality by assist requirements analysts to detect and resolvepossible ambiguity requirements. In future work, we will try to extend our work to convert requirements specification documents to UML diagrams.

\section{References}

[1] Basili, Victor R., Scott Green, Oliver Laitenberger, FilippoLanubile, Forrest Shull, SivertSorumgard. 1995. The Empirical Investigation of Perspective-Based Reading. Technical report the empirical investigation of perspective based reading(pp. 133-164).

[2] Nuseibeh, B., \& Easterbrook, S. 2000, May. Requirements engineering: a roadmap. In Proceedings of the Conference on the Future of Software Engineering (pp. 35-46).

[3] Belev, G. C. 1989, January. Guidelines for specification development.InReliability and Maintainability Symposium, ProceedingsIEEE, Annual (pp. 15-21).

[4] Christel, M. G., \& Kang, K. C. 1992. Issues in requirements elicitation (No.CMU/SEI-92-TR-12). CARNEGIE-MELLONUNIV PITTSBURGH PA SOFTWARE ENGINEERING INST.

[5] Donald G. Firesmith. 2007. Common Requirements Problems, Their Negative Consequences, and Industry Best Practices to Help Solve Them. In Journal of Object Technology, vol. 6, no. 1, JanuaryFebruary 2007, (pp. 17-33)

[6] Berry, D.M., Kamsties, E., Krieger, M.M.2003: From contract drafting to software specification: Linguistic sources of ambiguity, http://se.uwaterloo.ca/ ^dberry/handbook/ambiguityHandbook.pdf.

[7] Fabbrini, F., M. Fusani, S. Gnesi, and G. Lami. 2001. The Linguistic Approach to the Natural Language Requirements Quality: Benefit of the use of an Automatic Tool. SEW'01 proceeding of the 26th annual NASA Goddard Software En gineering Workshop, IEEE Computer Society Washington, DC, USA, 97.

[8] A. Fantechi, S. Gnesi, G. Lami, and A. Maccari,2003.Applications of Linguistic Techniques for Use CaseAnalysis," Requirements Engineering, vol. 8, (pp. 161-170).

[9] Nancy Ide and Jean Véronis, 1998, Introduction to the special issue on word sense disambiguation: The state of the art. Computational Linguistics - Special issue on word sense disambiguation, Volume 24 Issue 1, (pp. 2-40).

[10] Sri Fatimah Tjong, 2008, Avoiding ambiguity in requirements specifications.Thesis submitted to the University of Nottingham for the degree of Doctor of Philosophy.

[11] Sven Körner and TorbenBrumm, 2009, RESI-A natural language specification improver. IEEE International Conference on Semantic Computing (ICSC),(pp. 1-8).

[12] Sommerville, I. and Sawyer, P, 1997, Requirements engineeringA good practice guide. Chichester: John Wiley \& Sons Ltd. 